\title{
METALLICITY DISTRIBUTION AND KINEMATICS OF THE DISK AND HALO F-G STARS
}

\author{
ILFAN F. BIKMAEV \\ Special Astrophysical Observatory, Nizhnij Arkhyz, Karachai- \\ Circassian Republic, Russia, 357147, \\ e-mail: ibikm@sao.stavropol.su
}

The iron abundance of 160 disk and halo F-G-dwarfs is determined from 6-m spectra of 60 stars and published equivalent width data. Kurucz's (1979) model atmospheres and WIDTH6 programme are used for iron abundance determination. We have shown that FeI lines are formed under non-LTE conditions (Bikmaev et al.,1990), and so used Fell lines for iron abundance determination, following Bikmaev (1991). The use of the FeII lines and high quality homogeneous spectroscopic data decreases systematic and accidental errors and allows us to obtain qualitatively new results (Bikmaev,1994). The distribution of the sample of stars is a non-monotonous function of metallicity: at least 3 groups of stars are seen, corresponding to the three Galaxy populations - disk $([\mathrm{Fe} / \mathrm{H}]=+0.1$ to -0.3$)$, thick disk $([\mathrm{Fe} / \mathrm{H}]=-0.7$ to -0.8$)$ and halo $([\mathrm{Fe} / \mathrm{H}]=-1.3$ to -2.0$)$. In contrast with previous studies, a sharp boundary in the kinematic characteristics (the eccentricity of space orbits and angular velocity) was found at $[\mathrm{Fe} / \mathrm{H}]=-0.9$, the boundary between disk and halo stars. These kinematic properties demand a relatively long time between halo and disk formation. The average metallicity of the thick disk stars is very close to the metallicity of the Large Magellanic Cloud. Perhaps an explanation is merging during the approach of the LMC to the Galaxy several billion years ago.

\section{References}

Bikmaev, I.F., Bobritskij, S.S., Sakhibullin, N.A. (1990) Sov. Astron. Lett, 16, 213

Bikmaev, I.F. (1991) PhD Thesis, Nizhnij Arkhyz.

Bikmaev, I.F. (1994) ASP Conference Series, v.66 "Physics of the Gaseous and Stellar Disc of the Galaxy", ed. I.R.King, in press 